\title{
Late gadolinium enhancement is common in patients with hypertrophic cardiomyopathy and no clinical risk factors for sudden cardiac death: A single center experience
}

\author{
Kristopher S. Lyons ${ }^{1}$, Lana J. Dixon ${ }^{1}$, Nicola Johnston ${ }^{1}$, \\ Rebecca Noad ${ }^{1}$, Andrew Hamilton ${ }^{1}$, Nick McKeag ${ }^{1}$, Paul Horan ${ }^{2}$ \\ ${ }^{1}$ Cardiology Department, Royal Victoria Hospital Belfast, United Kingdom \\ ${ }^{2}$ Cardiology Department, Antrim Area Hospital, United Kingdom
}

\begin{abstract}
Background: Cardiac magnetic resonance (CMR) is used in the diagnosis and risk stratification of hypertrophic cardiomyopathy (HCM) and can detect myocardial replacement fibrosis (an independent predictor of adverse cardiac outcomes) using late gadolinium enhancement (LGE). Methods: We retrospectively analysed CMR studies carried out over a 2 year period identifying those which were diagnostic of HCM. 117 cases were analysed. Mean age of subjects was 53 years and 78 (67\%) were male. Mean ejection fraction (EF) was 68.3\% with a mean left ventricular (LV) mass index of $89.4 \mathrm{~g} / \mathrm{m}^{2}$. Hypertrophy was predominantly asymmetric in 94 (80\%).
\end{abstract}

Results: All subjects received gadolinium and 80 (68\%) had evidence of LGE. LVEF was lower (67 vs. $71 \% ; p=0.015$ ) and LV mass index higher (94 vs. $81 \mathrm{~g} / \mathrm{m}^{2} ; p=0.007$ ) in the LGE group. The proportion of patients with at least 1 clinical risk factor for sudden cardiac death (SCD) was similar in groups with and without LGE (48\% vs. $32 \% ; p=0.160)$. In this study, a significant proportion (62\%) of patients without clinical risk factors for SCD were found to have LGE on CMR. These patients would not currently be considered for therapy with an implantable cardiac defibrillator.

Conclusions: 1. Patients with HCM are at increased risk of SCD, but identifying patients who may benefit from implantable defibrillators is difficult. 2. LGE is associated with adverse cardiovascular outcomes in HCM, but is present in a large proportion of patients. 3. Many patients without clinical risk factors for SCD have LGE and would not currently be considered for an implantable cardiac device. (Cardiol J 2014; 21, 1: 29-32)

Key words: hypertrophic cardiomyopathy, cardiac magnetic resonance, sudden cardiac death, late gadolinium enhancement

\section{Introduction}

Cardiac magnetic resonance (CMR) is now widely used in the diagnosis and risk stratification of hypertrophic cardiomyopathy (HCM). It has several advantages over echocardiography including more accurate measurement of left ventricular function and mass, and it aids in the identification of apical HCM variants and alternative causes of hypertrophy such as athelets heart and Fabry's

Address for correspondence: Dr Kristopher S. Lyons, 160 Athelstan Road, Southampton, SO19 4DJ, tel: +44 7979898493, e-mail: kristopherlyons@hotmail.co.uk

Received: 03.03.2013 Accepted: 09.08.2013 


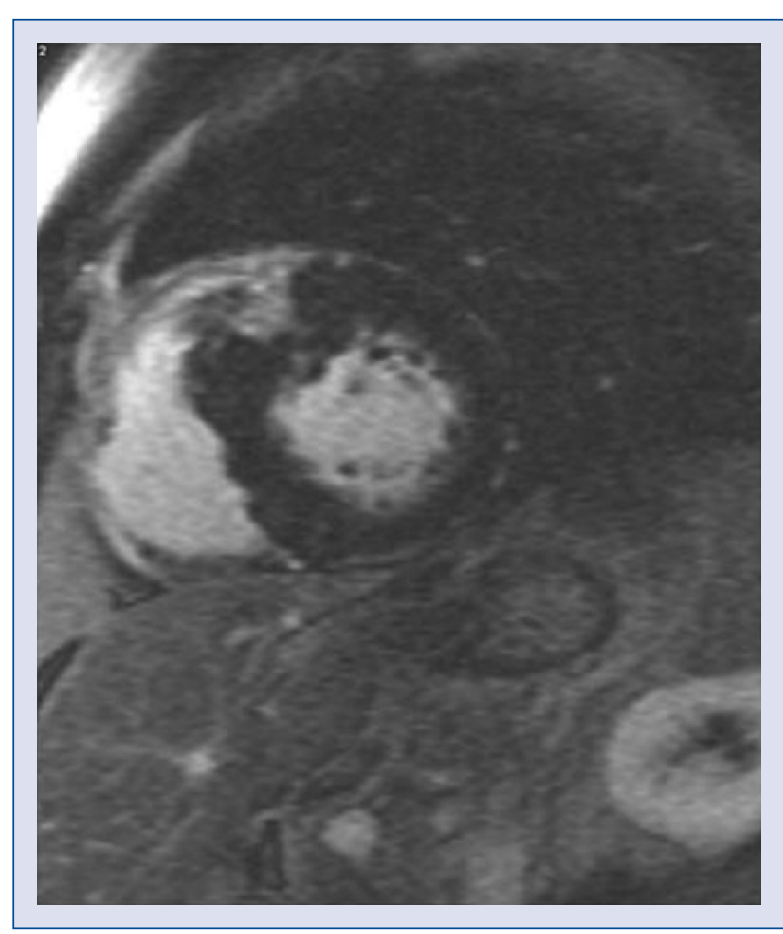

Figure 1. Late gadolinium enhancement within the interventricular septum in a patient with hypertrophic cardiomyopathy.

diseaes. Furthermore, it has the ability to demonstrate areas of myocardial replacement fibrosis using late gadolinium enhancement (LGE) (Fig. 1) which is increasingly being recognised as an independent predictor of adverse cardiac outcomes [1-6]. Several recently published reports have found an increased incidence of adverse cardiovascular events in patients with LGE compared to those without, and the sensitivity of clinical risk factors for prediction of sudden cardiac death (SCD) has been questioned. However previous studies have shown that LGE is present in a large proportion of patients with $\mathrm{HCM}$, and therefore identifies too large a population to be useful as a single predictor. We sought to look at the occurence of LGE in our population and assess the association of LGE with clinical risk factors.

\section{Methods}

We performed a retrospective analysis of all CMR studies carried out in a single center over a 24 month period from January 2010 to December 2011. Data was collected on all patients in whom CMR findings were consistent with a diagnosis of $\mathrm{HCM}$ ie. a hypertrophied and non-dilated ventricle in the absence of another condition capable of producing the observed degree of hypertrophy [7]. A cut-off for maximal wall thickness of $15 \mathrm{~mm}$ was used in adults in keeping with current guidelines although some patients with maximal wall thickness less than $15 \mathrm{~mm}$ were included if there was a strong clinical suspicion of HCM based on a family history of the condition in at least one first degree relative. Patients who had left ventricular (LV) hypertrophy which was potentially due to another condition such as hypertension were excluded, as were patients who did not receive a gadolinium based contrast agent as part of the imaging protocol.

All studies were performed on a Siemens Avanto 1.5 T scanner. LGE images were obtained after administration of intravenous gadobenate demeglumine $(0.2 \mathrm{mmol} / \mathrm{kg})$. LV function and mass were analysed after manual identification of endocardial and epicardial borders. The amount of LGE was not measured quantitively but was noted to be present or absent.

Patient demographics and occurence of clinical risk factors for SCD were obtained from review of clinical notes. CMR findings were taken from reports by level II or III accredited cardiologists. LV mass and function (indexed), distribution of hypertrophy and the presence of systolic anterior motion of the mitral valve (SAM) or elevated LV outflow tract (LVOT) velocities were noted.

As this was a retrospective analysis of patient records and no additional procedures beyond normal patient care were carried out, ethical approval and patient consent were not required.

\section{Statistical analysis}

Statistical analysis was carried out using SPSS v.17.0. Categorical variables were compared using $\chi^{2}$ test. Continuous variables were compared using the independent students $t$-test.

\section{Results}

One hundred and seventeen cases were included for analysis. Mean age of subjects was $53 \pm$ \pm 15.8 years and 78 (67\%) were male. Mean ejection fraction (EF) was $68.3 \pm 8.5 \%$ with a mean LV mass index of $89.4 \pm 26.7 \mathrm{~g} / \mathrm{m}^{2}$. SAM was present in $24(21 \%)$ and $16(14 \%)$ had an elevated LVOT velocity. The pattern of hypertrophy was predominantly asymmetric in $94(80 \%)$, predominantly apical in $13(11 \%)$ and concentric in $10(9 \%)$.

All subjects received gadolinium and some degree of LGE was present in $80(68 \%)$. There was no significant difference in prevalence of LGE based on gender $(\mathrm{p}=0.115)$. LGE was more common in apical (79\%) and concentric (80\%) patterns of hypertrophy compared to asymmetric (65\%) although this difference was not statistically significant 


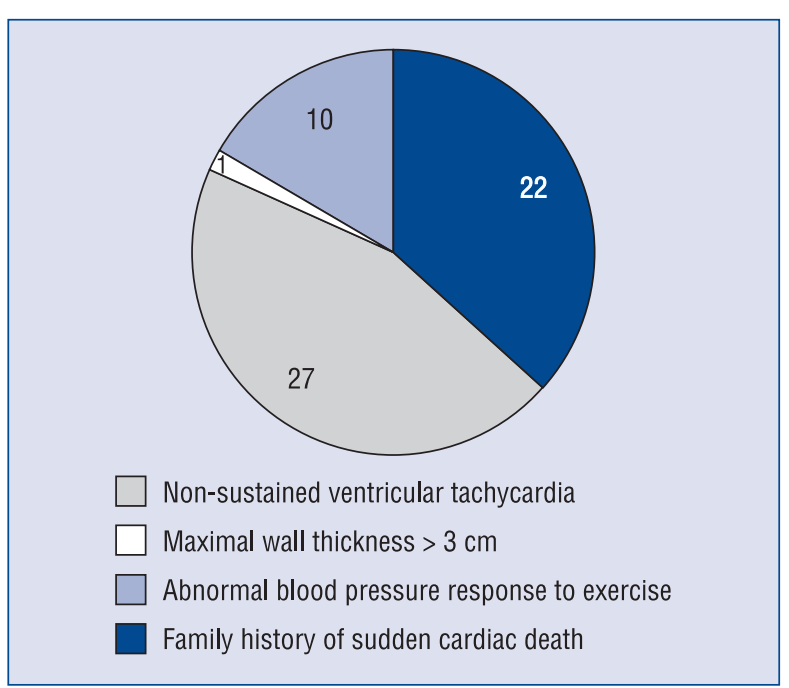

Figure 2. Frequency of individual clinical risk factors in the study group.

$(\mathrm{p}=0.254)$. LVEF was lower $(67 \pm 8.5 \%$ vs. $71 \pm$ $\pm 8.0 \% ; \mathrm{p}=0.015)$ and $\mathrm{LV}$ mass index higher $(94 \pm$ $\pm 27.9 \mathrm{~g} / \mathrm{m}^{2}$ vs. $\left.81 \pm 21.7 \mathrm{~g} / \mathrm{m}^{2} ; \mathrm{p}=0.007\right)$ in the LGE group.

Fifty (43\%) of the 117 patients had at least 1 clinical risk factor for SCD. The frequency of individual risk factors is shown in Figure 2. The percentage of patients with clinical risk factors in the LGE and no LGE groups is shown in Table 1. Only the difference in non sustained ventricular tachycardia (NSVT) between groups was statistically significant $(\mathrm{p}=0.035)$.

\section{Discussion}

The risk stratification of patients with $\mathrm{HCM}$ is complex. Current guidelines recommend that an implantable cardiac defibrillator be considered in patients with 1 or more clinical risk factors for $\mathrm{SCD}$ [7]. However there is mounting evidence that
LGE may be a much more specific predictor of poor prognosis in this condition, with adverse events being uncommon in patients without LGE [4]. The findings of this study are similar to previous reports, with regard to the proportion of patients with evidence of LGE [3]. In our group LGE was present in $68 \%$ of patients with HCM. However, when a risk factor is present in such a large proportion of patients, further discriminators are required to improve predictive power. These may include factors such as LV mass and quantification of LGE as a percentage of myocardial mass, with higher percentage correlating with greater risk [4].

Traditional risk factors for SCD were present in $50(43 \%)$ patients. Although NSVT was statistically more common in patients with LGE, overall the presence of any risk factor was not, suggesting that LGE is not simply a marker of commonly assessed clinical risk factors. A significant proportion of patients $(63 \%)$ without traditional clinical risk factors for SCD were found to have LGE on CMR and these patients would not currently be considered for therapy with an implantable cardiac defibrillator. The use of clinical risk factors in everyday practice presents several problems. Maximal wall thickness $>3 \mathrm{~cm}$ is present in a very small number of patients (only 1 in this study). Blood pressure response to exercise may be difficult to assess in many patients with mobility problems and can be unreliable in patients over 50 years of age [8]. Even family history of SCD may be difficult to quantify in patients who are unaware of their extended family tree. This study found that a significant proportion of patients with HCM who do not have clinical risk factors have evidence of LGE on CMR and may still be at increased risk of SCD. CMR with LGE provides a method of assessing myocardial replacement fibrosis and with futher modification may be the most sensitive means of identifying patients at risk for cardiovascular events.

Table 1. Occurrence of clinical risk factors for SCD according to presence or absence of LGE.

\begin{tabular}{lccc}
\hline & $\begin{array}{c}\text { Late gadolinium } \\
\text { enhancement }\end{array}$ & $\begin{array}{c}\text { No late gadolinium } \\
\text { enhancement }\end{array}$ & P \\
\hline 0 risk factors & $42(63 \%)$ & $25(37 \%)$ & 0.160 \\
$\geq 1$ risk factor & $38(48 \%)$ & $12(32 \%)$ & 0.160 \\
Family history of SCD & $17(21 \%)$ & $5(14 \%)$ & 0.447 \\
NSVT on Holter & $23(29 \%)$ & $4(11 \%)$ & 0.035 \\
Abnormal stress test & $7(9 \%)$ & $3(8 \%)$ & 1.000 \\
Maximal wall thickness $\geq 3 \mathrm{~cm}$ & $1(1 \%)$ & $0(0 \%)$ & 1.000 \\
\hline
\end{tabular}

LGE — late gadolinium enhancement; SCD — sudden cardiac death; NSVT — non sustained ventricular tachycardia 


\section{Limitations of the study}

This study has several limitations which must be considered. This is a retrospective analysis however for the purposes of assessing the relationship between LGE and clinical risk factors, a restrospective design was thought to be a reasonable approch. No endpoint data was therefore acquired (with regard to cardiovascular events in the population). The extent of LGE was assessed qualitatively as currently our CMR software does not allow quantitative assessment, however qualitative analysis may allow more accurate assessment of risk. The small numbers of patients in some groups made analysis difficult, and may have contributed to a lack of association with LGE and clinical risk factors over all however the number of subjects was reasonable for a single centre study of this type and the frequency of risk factors reflects that in a real world population.

\section{Conclusions}

1. Patients with HCM are at increased risk of SCD, but identifying patients who may benefit from implantable defibrillators is difficult.

2. LGE is associated with adverse cardiovascular outcomes in HCM, but is present in a large proportion of patients.

3. Many patients without clinical risk factors for SCD have LGE and would not currently be considered for an implantable cardiac device.

Conflict of interest: none declared

\section{References}

1. Hombach V, Merkle N, Bernhardt P, Rasche V, Rottbauer W. Prognostic significance of cardiac magnetic resonance imaging: Update 2010. Cardiol J, 2010; 17: 549-557.

2. Adabag A, Maron B, Appelbaum E et al. Occurrence and frequency of arrhythmias in hypertrophic cardiomyopathy in relation to delayed enhancement on cardiovascular magnetic resonance. J Am Coll Cardiol, 2008; 51: 1369-1375.

3. Green J, Berger J, Kramer C, Salerno M. Prognostic value of late gadolinium enhancement in clinical outcomes for hypertrophic cardiomyopathy. J Am Coll Cardiol Cardiovasc Imaging, 2012; 5: $370-377$.

4. O'Hanlon R, Grasso A, Roughton M et al. Prognostic significance of myocardial fibrosis in hypertrophic cardiomyopathy. J Am Coll Cardiol, 2010; 56: 867-874.

5. Bruder O, Wagner A, Jensen CJ et al. Myocardial scar visualized by cardiovascular magnetic resonance imaging predicts major adverse events in patients with hypertrophic cardiomyopathy. J Am Coll Cardiol, 2010; 56: 875-887.

6. Rubinshtein R, Glockner J, Ommen S et al. Characteristics and clinical significance of late gadolinium enhancement by contrast-enhanced magnetic resonance imaging in patients with hypertrophic cardiomyopathy. Circulation: Heart Failure, 2010; 3: 51-58.

7. 2011 ACCF/AHA Guideline for the Diagnosis and Treatment of Hypertrophic Cardiomyopathy: Executive Summary: A Report of the American College of Cardiology Foundation/American Heart Association Task Force on Practice Guidelines. Circulation 2011; 124: 2761-2796.

8. Olivotto I, Maron B, Montereggi A, Mazzuoli F, Dolara A, Cecchi F. Prognostic value of systemic blood pressure response during exercise in a community-based patient population with hypertrophic cardiomyopathy J Am Coll Cardiol, 1999; 33: 2044-2051. 